\title{
Cuerpo extraño intratorácico post traumatismo penetrante
}

\author{
ROBERTO GONZÁLEZ L.*, JUAN FARÍAS M.*, RODRIGO CAMPOS M.*, ENRIQUE SEGUEL S.*, \\ GLORIA ARZOLA G.**, DANIELA ALARCÓN P.* y EMILIO ALARCÓN C.*
}

\section{Thoracic foreign body after penetrating chest trauma}

We present the case of a 21 year old man with an intra thoracic foreign body after penetrating chest trauma. The foreign body was the blade of a knife. It was removed through the wound, without thoracotomy or video-assisted thoracic surgery (VATS) and the patient evolved without incidents. Intrathoracic foreign bodies secondary to penetrating trauma are rare. They are usually removed through thoracotomy or VATS. Both alternatives allow adequate exploration of the intra-thoracic structures and repair injuries that are potentially lethal. In stable patients and selected cases, they can be removed without surgery (without thoracotomy or VATS); always in an operating room and under general anesthesia, in case that surgical exploration could be needed after the procedure.

Key words: Chest injury, foreign bodies, penetrating wounds, wounds and injuries.

\section{Resumen}

Presentamos el caso de un hombre de 21 años con un cuerpo extraño intra torácico post traumatismo penetrante. El cuerpo extraño que correspondía a la hoja de un cuchillo, fue extraído a través de la misma herida traumática, sin toracotomía ni cirugía torácica video asistida (VATS). El paciente evolucionó favorablemente. Los cuerpos extraños intra torácicos secundarios a un traumatismo penetrante son infrecuentes. Se recomienda la extracción a través de toracotomía o de VATS, ambos procedimientos permiten una adecuada exploración de las estructuras intra torácicas y reparar potenciales lesiones. En pacientes estables y casos seleccionados se pueden extraer sin cirugía (sin toracotomía o VATS); siempre en pabellón quirúrgico y bajo anestesia general, teniendo todo preparado para cirugía en caso de ser necesario.

Palabras clave: Traumatismo torácico, cuerpo extraño, heridas penetrantes, heridas y traumatismos.

\section{Introducción}

Los traumatismos torácicos son responsables de aproximadamente el $25 \%$ de las muertes por traumatismos y contribuyen como un factor asociado mayor en otro $25 \%$ de muertes por lesiones traumáticas ${ }^{1,2}$.

La mayoría de los traumatismos penetrantes torácicos pueden ser manejados con un tubo pleural. Sólo entre el 10 a $15 \%$ de los traumatismos torácicos requieren toracotomía $a^{3,4}$.
Los cuerpos extraños intra torácicos secundarios a un traumatismo penetrante son infrecuentes y debido sus potenciales complicaciones en muchos casos se recomienda su extracción. Generalmente se extraen a través de una toracotomía o una cirugía torácica video asistida (VATS), ambas alternativas permiten una adecuada exploración de las estructuras intra torácicas y reparar lesiones potencialmente letales ${ }^{5-7}$.

Presentamos el caso de un hombre con un cuerpo extraño intra torácico post traumatismo

Hospital Regional "Dr. Guillermo Grant Benavente”, Departamento de Cirugía, Facultad de Medicina, Universidad de Concepción, Concepción, Chile:

* Equipo de Cirugía Cardiotorácica.

** Unidad de Urgencias. 
penetrante, que correspondía a la hoja de un cuchillo y en quien se extrajo el cuerpo extraño sin toracotomía ni VATS.

\section{Caso Clínico}

Hombre de 21 años, sin antecedentes mórbidos de importancia. Fue agredido por terceros con arma blanca en la vía pública. Ingresó al hospital aproximadamente dos horas después de la agresión. Presentaba una herida penetrante torácica derecha y una herida penetrante abdominal.

En la Unidad de Urgencias no presentó deterioro hemodinámico ni respiratorio. En el examen físico destacó la presencia de signos de irritación peritoneal. La radiografía de tórax mostró un cuerpo extraño intra torácico compatible con la hoja de un cuchillo (Figura 1). La tomografía computada de tórax demostró que la hoja del cuchillo presentaba un segmento intra pulmonar y que el vértice de ésta, se encontraba aproximadamente a 2 milímetros del hilio pulmonar derecho (Figura $2 \mathrm{~A}$ ), además, se observó un hemotórax leve (Figura 2 B). El equipo tratante en la Unidad de Urgencias, decidió la extracción del cuerpo extraño intra torácico y cirugía abdominal.

\section{Procedimiento quirúrgico}

En el pabellón quirúrgico y con anestesia general, se extrajo la hoja del cuchillo a través de la herida traumática torácica ( $\sin$ toracotomía o VATS) y se instaló un tubo pleural. Se observó por algunos minutos el débito por el tubo pleural y se decidió el inicio de la cirugía abdominal. En el abdomen se realizó una laparotomía explora-

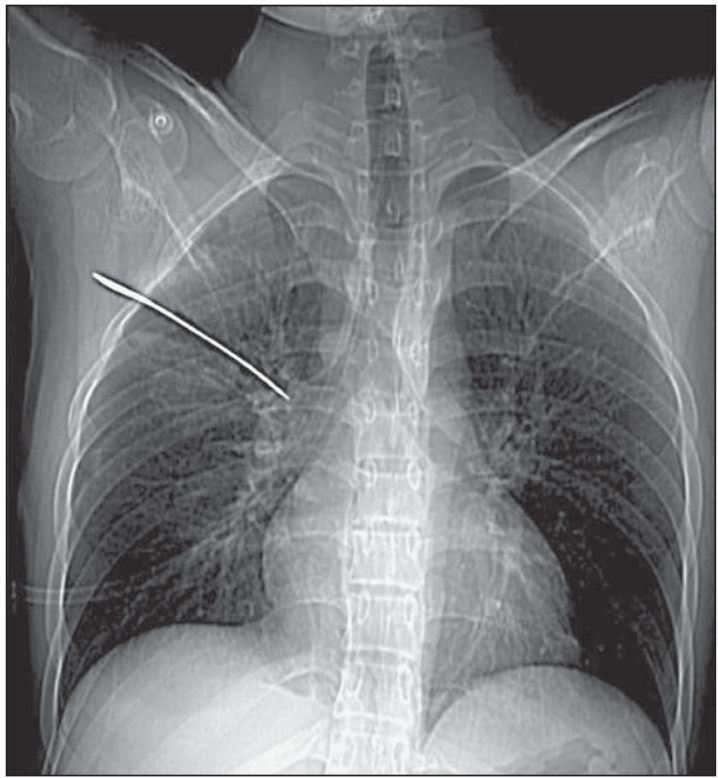

Figura 1. Radiografía de tórax que muestra cuerpo extraño intra-torácico (hoja de cuchillo).

dora, presentaba una lesión de colon transverso que se suturó, no se encontró otra lesión intra abdominal.

\section{Evolución post operatoria}

En el post operatorio, el caso fue conocido y tratado por el Equipo de Cirugía Cardiotorácica. Evolucionó favorablemente con expansión pulmonar completa y débito bajo por el tubo pleural. El tubo de drenaje pleural se mantuvo por 5 días.

Fue dado de alta al octavo día post operado con una evolución favorable, sin ocupación pleu-

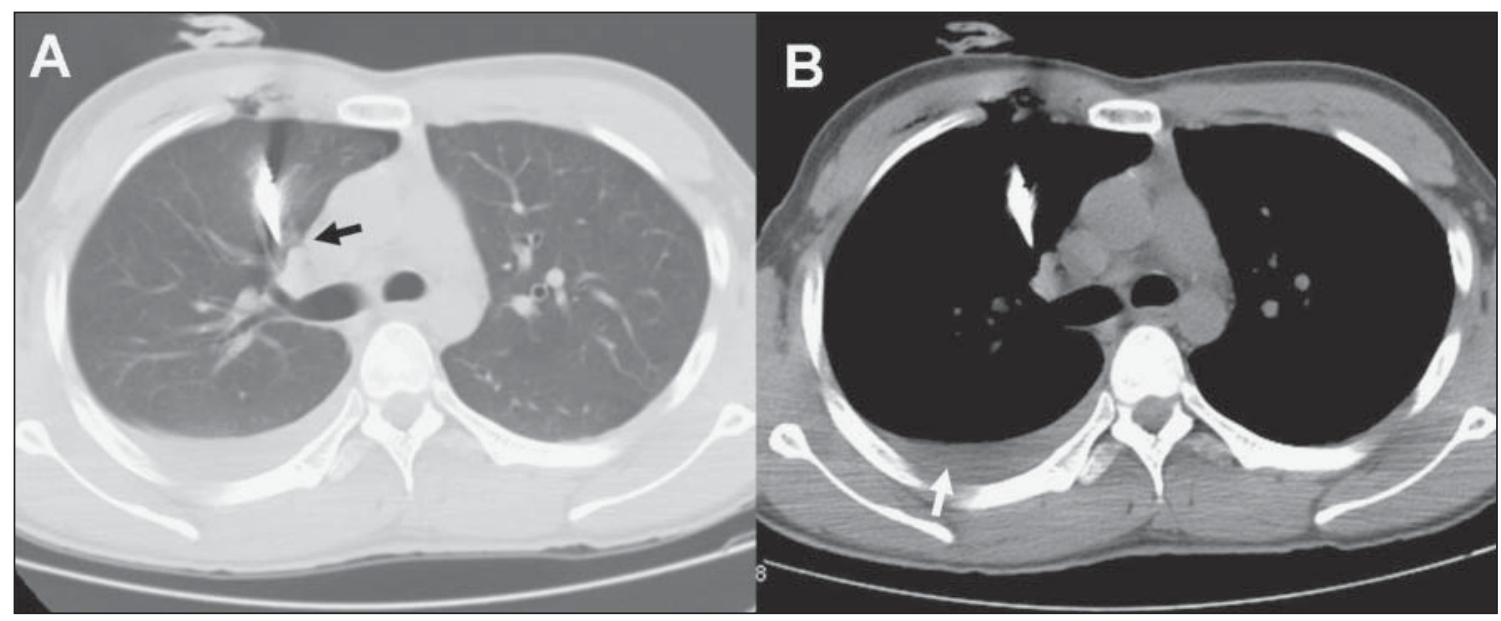

Figura 2. Tomografía computada de tórax que muestra relación entre hoja de cuchillo e hilio pulmonar derecho (flecha negra, Figura 2A) y hemotórax leve (flecha blanca, Figura 2B). 
ral y asintomático desde el punto de vista abdominal. A los dieciocho meses de seguimiento se encuentra asintomático y la radiografía de tórax no presenta hallazgos significativos.

\section{Discusión}

Las comunicaciones sobre cuerpos extraños intra torácicos post traumatismo son sorprendentemente escasas. Generalmente corresponden a casos aislados, a diversos tipos de cuerpo extraño (fragmentos de vidrio, trozos de madera, hojas de cuchillo, clavos de carpintería, etc) y se presentan asociados a diversos mecanismos traumáticos, por lo que no existe consenso en relación a su tratamiento ${ }^{5-7}$.

Weissberg y cols, en una revisión de la experiencia por más de 30 años en su centro, presen$\tan 22$ casos de cuerpos extraños pleurales y en pared torácica, de los cuales sólo 5, corresponden a casos secundarios a traumatismos?

Debido a que se pueden asociar a otras lesiones y por sus potenciales complicaciones (como migración e infección) en muchos casos se recomienda la extracción. Algunos objetos pequeños, romos, periféricos o con mucho tiempo de evolución sin evidencias de complicaciones, podrían no ser extraídos ${ }^{5-7}$.

El abordaje a través de una toracotomía, tradicionalmente ha sido el enfrentamiento para la extracción de los cuerpos extraños intratorácicos. La toracotomía permite una adecuada exposición para la extracción del cuerpo extraño bajo visión directa y para reparar potenciales lesiones asociadas ${ }^{7}$.

Hoy en día la cirugía mínimamente invasiva o cirugía torácica video asistida (VATS) constituye uno de los abordajes más utilizados en el diagnóstico y tratamiento de un amplio espectro de patologías torácicas. Las indicaciones más frecuentes de VATS en lesiones traumáticas son hemotórax retenido o coagulado, neumotórax persistente y evaluación de lesiones diafragmáticas. Además, la VATS ha demostrado ser segura y efectiva en pacientes con traumatismos evitando un importante número de toracotomías y laparotomías ${ }^{8,9}$.

Williams y cols, han comunicado tres casos de cuerpos extraños post traumatismo penetrante que fueron extraídos por VATS exitosamente. Además de las ventajas propias de los procedimientos mínimamente invasivos, destacan que la VATS permite una adecuada exploración de la pared torácica, pulmón, mediastino y diafragma, señalando que en pacientes estables corresponde al abordaje de primera elección para la extracción de cuerpos extraños post traumáticos ${ }^{6}$.

Una alternativa en pacientes estables, es la extracción del cuerpo extraño sin cirugía (sin toracotomía o VATS), esto debe ser bien evaluado con imágenes adecuadas, en que se observe el cuerpo extraño y su relación con estructuras intratorácicas. Siempre debe ser en el pabellón quirúrgico y bajo anestesia general; todo debe estar preparado para la cirugía en caso de ser necesario, ya que se pueden presentar complicaciones como hemorragias que pueden poner en riesgo la vida del paciente ${ }^{7}$.

En el caso presentado, como el paciente además tenía indicada una laparotomía exploradora, el equipo tratante de la Unidad de Urgencias decidió la extracción del cuerpo extraño a través de la propia herida traumática en el pabellón quirúrgico y con anestesia general; se extrajo el cuerpo extraño, se observó el débito del tubo pleural y se consideró que al no existir evidencia de una complicación intratorácica la exploración abdominal debía realizarse a la brevedad.

La evolución de este paciente fue muy favorable, sin complicaciones precoces y ni tardías en el seguimiento de dieciocho meses. Sin embargo, como Equipo de Cirugía Cardiotorácica, creemos que la primera opción en este tipo de casos (principalmente por el tamaño del cuerpo extraño, trayecto y cercanía con el hilio pulmonar) es la extracción mediante VATS o toracotomía, dependiendo de la urgencia, disponibilidad o experiencia del equipo quirúrgico.

Los cuerpos extraños intratorácicos post traumatismo penetrante son infrecuentes y en general se recomienda su extracción. La VATS o la toracotomía, dependiendo de cada caso en particular, son los abordajes recomendados. En casos excepcionales se puede realizar la extracción sin cirugía (sin toracotomía o VATS), siempre en pabellón quirúrgico, con anestesia general y todo debe estar preparado para la cirugía en caso de ser necesario.

\section{Bibliografía}

1.- MEREDITH J W, HOTH J J. Thoracic trauma: When and how to intervene. Surg Clin N Am 2007; 87: 95118.

2.- MATTOX K L, WALL M J JR. Newer diagnostic measures and emergency management. Chest Surg Clin N Am 1997; 7: 213-26.

3.- BASTOS R, BAISDEN C, HARKER L, CALHOON J H. Penetrating thoracic trauma. Semin Thorac Cardiovasc Surg 2008; 20: 19-25. 
4.- PHELAN H A, PATTERSON S G, HASSAN M O, GONZALEZ R P, RODNING C B. Thoracic damagecontrol operation: principles, techniques, and definitive repair. J Am Coll Surg 2006; 203: 933-41.

5.- BURACK J H, AMULRAJ EA, O'NEILL P, BREVETTI G, LOWERY R C. Thoracoscopic removal of a knife impaled in the chest. J Thoracic Cardiovasc Surg 2005; 130: 1213-4.

6.- WILLIAMS C G, HAUT E R, OUYANG H, RIALL T S, MAKARY M, EFRON DT, et al. Video-Assisted
Thoracic surgery removal of foreign bodies after penetrating chest trauma. J Am Coll Surg 2006; 202: 848-52.

7.- WEISSBERG D, WEISSBERG-KASAV D. Foreing Bodies in pleura and chest wall. Ann Thorac Surg 2008; 86: 958-61.

8.- AHMED N, JONES D. Video-assisted thoracic surgery: state of the art in trauma care. Injury 2004; 35 : 479-89.

9.- REDY V S. Minimally invasive techniques in thoracic trauma. Semin Thorac Cardiovasc Surg 2008; 20: 72-7.

Correspondencia a:

Dr. Roberto González Lagos

Departamento de Cirugía, Facultad de Medicina

Universidad de Concepción.

Janequeo esquina Chacabuco, Concepción, Chile.

Fono-Fax: 56-41-2204881

E-mail: rgonzalezlagos@udec.cl 